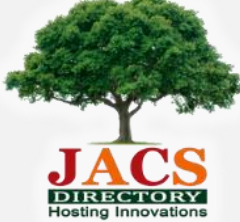

\title{
Investigation on the Properties of Pure and Strontium Doped NiO Nanoparticles by Co- Precipitation Method
}

\author{
R. Thanal1,*, S. Sasikala², M. Bakiyalakshmi ${ }^{1}$ \\ ${ }^{1}$ Department of Physics, Gonzaga College of Arts and Science for Women, Kirshnagiri - 635 108, Tamilnadu, India. \\ ${ }^{2}$ Department of Physics, Kamban College of Arts and Science for Women, Tiruvannamalai - 606 603, Tamilnadu, India.
}

\section{ART IC LE DETAILS}

Article history:

Received 17 May 2018

Accepted 07 June 2018

Available online 08 June 2018

\section{Keywords:}

Strontium

Nickel Oxide

Co-Precipitation Method

\begin{abstract}
A B S T R A C T
Pure and strontium doped nickel oxide nanoparticles were synthesized by co-precipitation method and calcinated at $400^{\circ} \mathrm{C}$ from nickel chloride as precursor agent and strontium chloride as doping agent. The samples have been studied by various characterization techniques such as UV-visible, FTIR, XRD and FESEM. The crystallite size and structure of $\mathrm{NiO}$ nanoparticles were calculated from XRD pattern. The morphology and particles shape were analyzed by FESEM studies. The SPR absorption peak and band gap value were calculated by UV-visible absorption spectra.
\end{abstract}

\section{Introduction}

An inorganic nickel oxide nanoparticles has been received great attention because of its unique electrical, magnetic and catalytic properties compare to bulk material [1] and $\mathrm{NiO}$ shows p-type semiconducting nanomaterial with a wide range of band gap value (3.6$4.0 \mathrm{eV}$ ) and this leads to a great applications in various field due to their above mentioned properties such as electrochromic films [2], catalysts [3], solar cell, lithium batteries [4] and gas sensor [5]. Several methods have been proposed to prepare nickel oxide nanoparticle such as simple liquid phase process [6], thermal decomposition route [7], sol-gel method [8] and sonochemical method [9]. Among these methods, co-precipitation method has a clear advantages including simplicity, low cost, easy procedure and produce a mass collection of samples. The size, morphology and shape of nanoparticles depends on the synthesis method, precursor concentration, surfactant and calcination temperature.

In this present work, pure and strontium doped nickel oxide nanoparticles preparation by co-precipitation method and their material properties have been discussed.

\section{Experimental Methods}

\subsection{Materials}

All chemicals used in this work were analytical grade reagents and used without further purification. Nickel chloride, sodium dodecyl sulfate, strontium chloride and sodium hydroxide $(\mathrm{NaOH})$ were purchased from Merck Company. Deionized water was used in this present work.

\subsection{Preparation of Pure and Strontium Doped Nickel Oxide Nanoparticles}

The required amount of nickel chloride, sodium dodecyl sulfate, strontium chloride and sodium hydroxide were dissolved separately in double distilled water and stirred it for 30 minutes. Then sodium dodecyl sulphate solution was added into nickel chloride and this mixture was stirred for 30 minutes. Then sodium hydroxide solution was added drop by drop into the mixture of solution and color of the solution was changed from deep green to light green as stirring was continued for three hours. A gel like green colour precipitate appeared at the bottom of beaker. The

precipitate was washed several times with distilled water and ethanol. The precipitate was dried at $80^{\circ} \mathrm{C}$ using a hot air oven. Then final product was calcinated at $400{ }^{\circ} \mathrm{C}$ for 3 hour using muffle furnace. A similar procedure was followed to prepare strontium doped nickel oxide particles. Strontium solution was added into the mixture before adding sodium hydroxide solution. The final solution was stirred and the precipitate was washed and dried. Then sample was calcinated at $400^{\circ} \mathrm{C}$ for 3 hour using muffle furnace.

\subsection{Characterizations}

UV-visible absorption spectra were carried out using Lambda 35 UVvisible spectrophotometer in the wavelength range $300-800 \mathrm{~nm}$. FTIR characterization is analyzed in the spectral range from $400-4000 \mathrm{~cm}^{-1}$ using Shimadzu Fourier transform infrared spectrometer. The powder XRD pattern was carried out by Enraf Nonius CAD4-F diffractometer with the CuKa $(\lambda=1.540 \AA)$ radiation. The field emission scanning electron microscopy characterization was taken out by FESEM quanta 20 spectrophotometer.

\section{Results and Discussion}

\subsection{Optical Analysis}

Fig. 1 shows UV-visible absorption spectra of pure and strontium doped nickel oxide nanoparticles.

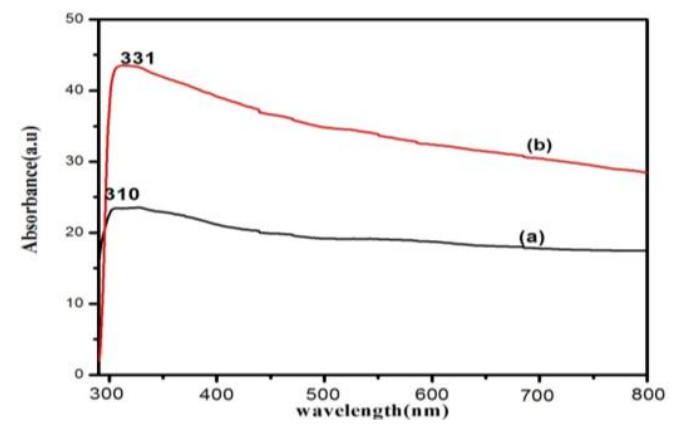

Fig. 1 UV-vis. spectra of (a) pure and (b) strontium doped nickel oxide nanoparticle

The pure and strontium doped NiO nanoparticles shows SPR absorption peak maximum at $310 \mathrm{~nm}$ and $331 \mathrm{~nm}$ respectively. The spectrum 
obtained due to optical absorption can be analyzed by the energy band gap of the semiconductor nanomaterials and its calculated by this simple energy equations, $E=h c / \lambda$, where $h$ is the Planck's constant, $c$ is the light velocity and $\lambda$ is the wavelength of absorbed peak. The energy band gap values calculated was found to be $4.00 \mathrm{eV}$ for pure $\mathrm{NiO}$ nanoparticles and $3.7 \mathrm{eV}$ for strontium doped nickel oxide nanoparticles respectively.

\subsection{Functional Group Analysis}

Fig. 2 shows FTIR spectra of pure and strontium doped nickel oxide nanoparticles by co-precipitation method. The peaks appear at $3366 \mathrm{~cm}^{-1}$ was assigned to $\mathrm{OH}$ stretching mode of vibration and this peaks reveal that water was absorbed on metal oxide surface [10]. The peaks absorbed at $1619 \mathrm{~cm}^{-1}$ was correspond to $\mathrm{H}-\mathrm{O}-\mathrm{H}$ bending mode of vibration. Then peaks absorbed at $1113 \mathrm{~cm}^{-1}$ represents the $0-C=0$ symmetric stretching mode of vibration. The peaks appear at $893 \mathrm{~cm}^{-1}$ was assigned to $\mathrm{N}-\mathrm{H}$ stretching vibration and after this peak we could clearly see that strontium doped $\mathrm{NiO}$ peaks were slightly shifted from pure $\mathrm{NiO}$ peaks. This shifted peak was due to strontium as a doping agent on metal oxide nanoparticles. The peaks absorbed at $607 \mathrm{~cm}^{-1}$ and $471 \mathrm{~cm}^{-1}$ were assigned to Ni-O mode of vibration [11] and this confirms that pure $\mathrm{NiO}$ compound was successfully formed. The peak absorbed at $445 \mathrm{~cm}^{-1}$ was assigned to $\mathrm{Sr}-\mathrm{Ni}$ 0 stretching mode of vibration.

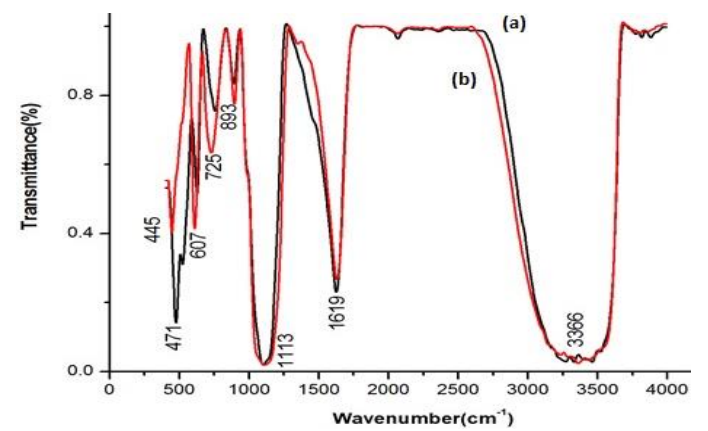

Fig. 2 FTIR spectra of nickel oxide nanoparticles (a) pure (b) strontium doped

\subsection{Structural Analysis}

Fig. 3 shows the powder XRD pattern of pure and strontium doped nickel oxide nanopowder. The samples were calcinated at a temperature $400{ }^{\circ} \mathrm{C}$. The diffraction peaks (003), (202) and (021) correspond to rhombohedral structure of nickel oxide with lattice parameter $\mathrm{a}=\mathrm{b}=2.954$ and $\mathrm{c}=7.226 \AA$ and it is good agreement with the reported values (JCPDS Card 89-7101). No additional peaks of other phases have been found in XRD and this confirms that the formation of pure rhombohedral structure of nickel oxide. In the strontium doped nickel oxide diffraction pattern, there was small peak shifts in the lower $2 \theta$ angle respect to pure nickel oxide was observed.

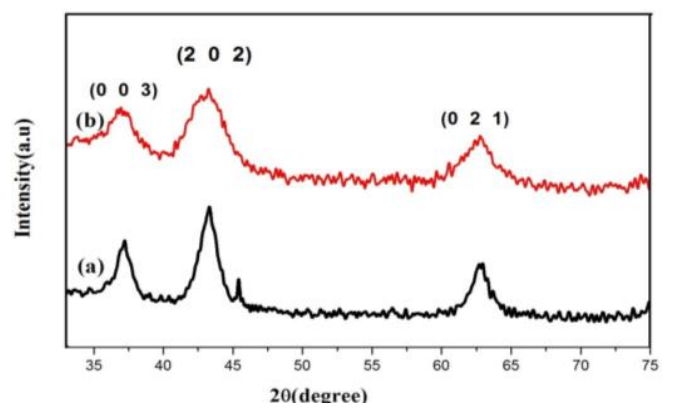

Fig. 3 XRD patterns of nickel oxide nanoparticles (a) pure (b) strontium doped

The crystallite sizes were calculated using Debye Scherrer's equation, $D=k \lambda / \beta \cos \theta$, where, $d$ is particle size in nanometer, $\lambda$ is wavelength of the radiation, $\mathrm{k}$ is constant equal to $0.94, \beta$ is the full width at maximum intensity and $\theta$ is peak position. The crystallite sizes of the pure and strontium doped nickel oxide nanoparticles size were found to be around $6.7 \mathrm{~nm}$ and $4.3 \mathrm{~nm}$ respectively.

\subsection{Morphological Analysis}

Fig. 4 shows FESEM images of pure and strontium doped $\mathrm{NiO}$ nanoparticles calcinated at $400{ }^{\circ} \mathrm{C}$. From this image it is observed that pure $\mathrm{NiO}$ nanoparticle shows cluster like morphology along with some agglomerations exist between the nanoparticles. Strontium doped $\mathrm{NiO}$ sample exhibits non-uniform spherical shape and random distributions were observed. Thus dopant can control the particle size and also avoid the particles agglormeration than pure metal oxide nanoparticles.
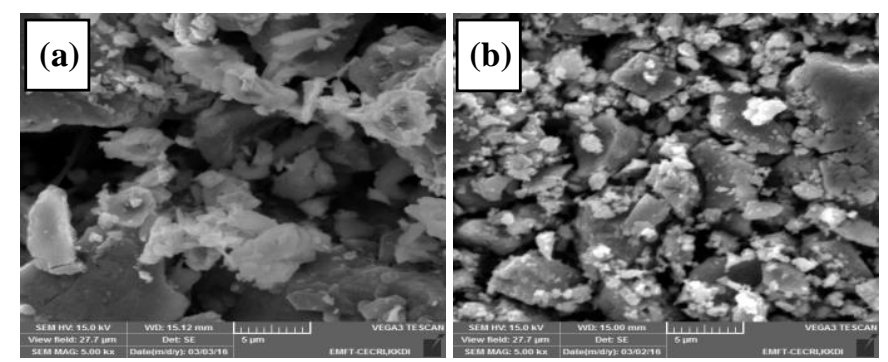

Fig. 4 FESEM images of nickel oxide nanoparticles (a) pure (b) strontium doped

\section{Conclusion}

Pure and strontium doped nickel oxide nanoparticles have been successfully synthesized by simple co-precipitation method. The band gap energy has been evaluated for pure nickel oxide and $\mathrm{Sr}$ doped nickel oxide and the values are found to be $4.0 \mathrm{eV}$ at and $3.7 \mathrm{eV}$ respectively. The XRD results show a slight shift in the peak positions due to doping agent, however structure of crystallites remain the same and also crystallite size of strontium doped nickel oxide nanoparticles are smaller than pure nickel oxide nanoparticles. The surface morphology of pure nickel oxide and strontium doped nickel oxide exhibit cluster like morphology along with some agglomerations.

\section{References}

[1] L.G. Teoh, K.D. Li, Synthesis and characterization of NiO nanoparticles by sol gel method, Mater. Trans. 53 (2012) 2135-2140.

[2] J.L. Garcia-Miquel, Q. Zhang, S.J. Allen, A. Rougier, A. Blyr, H.O. Davies, Nickel oxide sol-gel films from nickel diacetate for electrochromic applications, Thin Solid Films 424 (2003) 165-170.

[3] S.V. Ganachari, R. Bhat, R. Deshpande, Venkataraman, Synthesis and characterization of nickel oxide nanoparticles by self-propagating low temperature combustion method, Recent Res. Sci. Technol. 4 (2012) 50-53.

[4] N.N.M. Zorkipli, N.H.M. Kaus, A.A. Mohamad, Synthesis of NiO nanoparticles through Sol-gel method, Proc. Chem. 19 (2016) 626-631.

[5] K.M. Dooley, S.Y. Chen, J.R.H. Ross, Stable Nickel-containing catalysts for the oxidative coupling of methane, J. Catal. 145 (1994) 402-408.

[6] A. Rahdar, M. Aliahmad, Y. Azizi, NiO nanoparticles: synthesis and characterization, Jour. Nanosci. 5 (2015) 145-151.

[7] N.M. Hosny, Synthesis, characterization and optical band gap of $\mathrm{NiO}$ nanoparticles derived from anthranilic acid precursors via a thermal decomposition route, Polyhedron 30 (2011) 470-476.

[8] L. Xiang, X.Y. Deng, Y. Jin, Experimental study on synthesis of NiO nanoparticles, Scr. Mater. 47 (2002) 219-224.

[9] M. Mohammadijoo, Z. Naderi Khorshidi, S.K. Sadrnezhaad, V. Mazinani, Synthesis and characterization of nickel oxide nanoparticle with wide band gap energy prepared via thermochemical processing, Nanosci. Nanotechnol. Int. J. 4 (2014) 6-9.

[10] G.J. Li, S. Kawi, Synthesis, characterization and sensing application of novel semiconductor oxides, Talanta 45 (1998) 759-766.

[11] F. Davar, Z. Fereshteh, M. Salavati-Niasari, Nanoparticles Ni and NiO: Synthesis, characterization and magnetic properties, J. Alloys Compd. 476 (2009) 797801. 